MIDPI sciforum
MOL2NET, International Conference Series on Multidisciplinary Sciences

Conference on Molecular, Biomedical, and Computational Sciences and Engineering, 7th ed.

\title{
Antipyretic effect of myrcene (MCN) as a potential therapeutic alternative for the treatment of systemic inflammation response (SI) in euthermic rats model
}

Maycon Tavares Emílio-Silva ${ }^{a}$, Melina Luzzi Zarricueta ${ }^{a}$, Vinicius Peixoto Rodrigues ${ }^{a}$, Priscila Romano Raimundo ${ }^{a}$, Lúcia Regina Machado da Rocha ${ }^{a}$, Clélia Akiko Hiruma-Lima ${ }^{a}$.

${ }^{a}$ Department of Structural and Functional Biology (Physiology), Institute of Biosciences, São Paulo State University, Botucatu 18618970, São Paulo, Brazil.

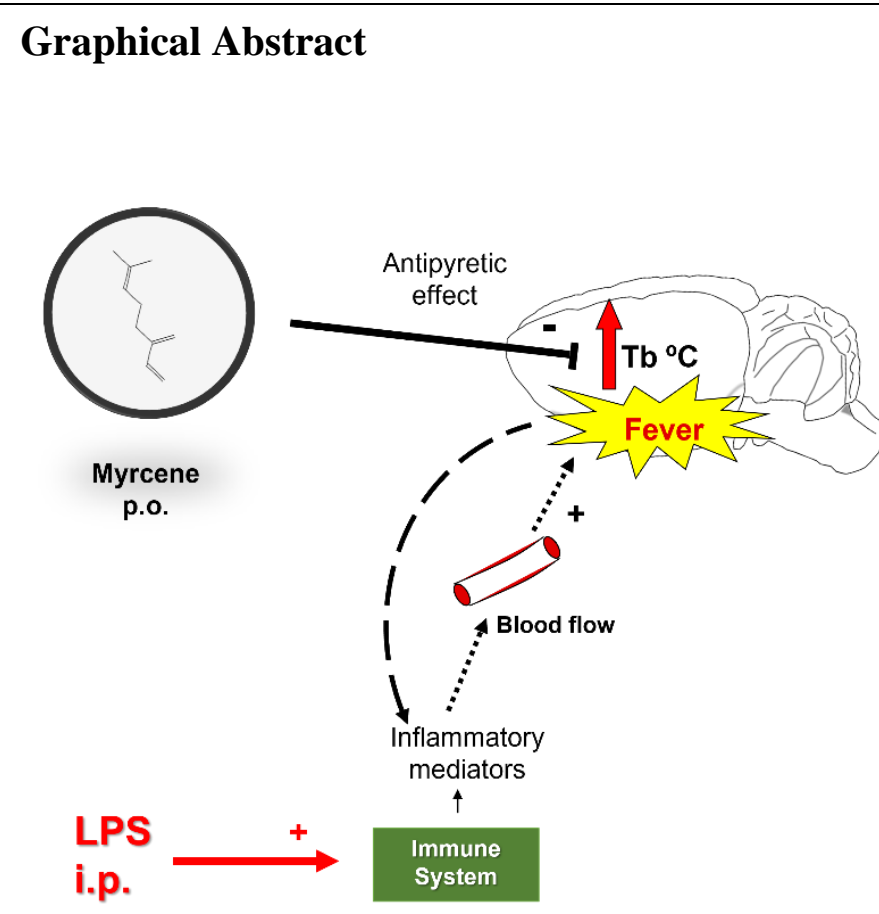

\section{Abstract.}

The new coronavirus pneumonia (COVID-19) is characterized by hemodynamic changes with a large release of inflammatory mediators, that inducing a systemic inflammation response (SI). Thus, SI can be induced by peripheral injection of lipopolysaccharide (LPS) in rodents, which promote the increase of systemic inflammatory mediators that induced an essential survival effector response, such as fever. Myrcene (MCN) is a monoterpene with anti-inflammatory, antioxidant, and analgesic actions are described. Our aim was to evaluate the MCN effect in euthermic rats against SI induced by low-dose LPS. Male Wistar rats were used to assess body temperature $(\mathrm{Tb})$ by dataloggers. The animals were orally treated with Tween $80-1 \%$ (Tw; 10 $\mathrm{mL} / \mathrm{kg})$ or $\mathrm{MCN}(7.5 \mathrm{mg} / \mathrm{kg}) 30 \mathrm{~min}$ before the i.p. administration of induction with LPS (100 $\mu \mathrm{g} / \mathrm{kg}$ ) or Saline $(1 \mathrm{~mL} / \mathrm{kg})$. After $360 \mathrm{~min}$, the rats were sacrificed to evaluate the inflammatory mediators' levels through Multiplex assay. Rats in the Tw+LPS group present a significant increase in $\mathrm{Tb}$ when compared to the control group ( $\mathrm{Tw}+$ Saline). The MCN treatment was 
able to significantly reduce $\mathrm{Tb}$, demonstrated an antipyretic effect during SI-induced LPS. When investigating the inflammatory signaling caused by LPS administration, the demonstrated a significant increase in serum levels of MIP-1 $\alpha$, MCP-1, IP-10, TNF- $\alpha$, Fractalkine, IL-10, and IL-5. However, our treatment with MCN is not involved in peripheral signaling of inflammatory mediators during SI.

\section{Introduction}

The pandemic of new coronavirus pneumonia (COVID-19) is of great concern worldwide, due to the millions of deaths caused in the last year. COVID-19 is characterized by hemodynamic changes, such as venous thromboembolism and intravascular coagulation. In addition to a large release of inflammatory mediators, such as chemoattractant monocyte protein (MCP)-1, inflammatory macrophage protein 1- $\alpha$ (MIP1 $\alpha$ ), interferon gamma-inducible protein (IP)-10, that inducing a systemic inflammation response (SI) [1]. Thus, an important experimental model of SI is the peripheral injection of lipopolysaccharide (LPS) in rodents, which promote the production and release of peripheral inflammatory mediators [tumor necroses factor (TNF)- $\alpha$, interleukin (IL)-5, IL-10 and fractalkine]. This inflammatory signaling promotes essential survival effector responses, such as an increase in deep body temperature ( $\mathrm{Tb}$ ), also known as fever [2-4]. The search for new drugs that alleviate this condition can alleviate in SI treatment, mainly, during COVID-19. Myrcene (MCN) is a monoterpene present in the Citrus aurantium essential oil, and their healing, anti-ulcer, anti-inflammatory, antioxidant, and analgesic actions are described in the literature [5]. However, the effect of this monoterpene during SI is unknown. Aim: To evaluate the effect of MCN in euthermic rats against systemic inflammation induced by lowdose LPS.

\section{Materials and Methods}

Male Wistar rats (5-6 weeks, 230-280g, $\mathrm{n}=7-8$ ) were used to assess Tb by dataloggers implanted in the abdominal cavity [6]. The rats were orally treated with vehicle - Tween $80-1 \%$ (Tw; $10 \mathrm{~mL} / \mathrm{kg}$ ) or MCN $(7.5 \mathrm{mg} / \mathrm{kg}) 30 \mathrm{~min}$ before the i.p. administration of induction with LPS $(100 \mu \mathrm{g} / \mathrm{kg})$ or Saline $(1$ $\mathrm{mL} / \mathrm{kg}$ ). After $360 \mathrm{~min}$ of LPS injection, the rats were sacrificed to collected of serum to evaluate the inflammatory mediators' levels (MIP-1 $\alpha$, MCP-1, IP-10, TNF- $\alpha$, Fractalkine, IL-10 and IL-5) through Multiplex (Merck Milliplex; Millipore Corporation, Billerica, MA, USA) assay. Statistical significance was determinate by ANOVA followed Tukey post-hoc test $(\mathrm{p}<0.05)\left(\right.$ CEUA-IBB/UNESP $\mathrm{n}^{\circ}$ 8259150621).

\section{Results and Discussion}

Rats in the Tw+LPS group present a significant increase in Tb when compared to the control group $(\mathrm{Tw}+$ Saline $)\left(\mathrm{Tw}+\mathrm{Saline}=53.42 \pm 5.42^{\circ} \mathrm{C} \mathrm{x} \min v s\right.$. Tw $\left.+\mathrm{LPS}=197.4 \pm 12.6^{\circ} \mathrm{C} \mathrm{x} \mathrm{min}.\right)$. However, the MCN treatment was able to significantly reduce $\mathrm{Tb}$ when compared with the Tw+LPS group $\left(\mathrm{Tw}+\mathrm{LPS}=197.4 \pm 12.6^{\circ} \mathrm{C} \times \mathrm{min} v s . \mathrm{MCN}+\mathrm{LPS}=137.1 \pm 15.6^{\circ} \mathrm{C} \times \mathrm{min}.\right)$, indicating an antipyretic effect of MCN during SI. When investigating the inflammatory signaling caused by LPS administration, the Tw+LPS group showed a significant increase in serum levels of MIP-1 $\alpha$, MCP-1, IP-10, TNF- $\alpha$, Fractalkine, IL-10, and IL-5, when compared to the control group ( $\mathrm{p}$ < 0.05). These factors are directly 
related to thrombosis events and indicators of the systemic inflammatory response during COVID-19. However, our treatment with MCN did not act in the modulation of peripheral signaling to reduce the serum levels of inflammatory mediators, when compared with the Tw+LPS group ( $p>0.05)$.

\section{Conclusions}

Our data demonstrate that MCN has an antipyretic effect after 360 min LPS administration. However, MCN was not able to reduce inflammatory signaling during SI. Possibly, the monoterpene effect may be involved in central nervous system mechanisms during the inflammatory response caused by LPS injection.

\section{References}

.1. Chen, Y.; Wang, J.; Liu, C.; Su, L.; Zhang, D.; Fan, J.; Yang, Y.; Xiao, M.; Xie, J.; Xu, Y.; et al. IP-10 and MCP-1 as biomarkers associated with disease severity of COVID-19. Mol. Med. 2020, 26, 1-12, doi:10.1186/S10020-020-00230-X/FIGURES/5.

2. Blatteis, C.M.; Sehic, E. Cytokines and fever. Ann. N. Y. Acad. Sci. 1998, 840, 608-18.

3. Garami, A.; Steiner, A.A.; Romanovsky, A.A. Fever and hypothermia in systemic inflammation. In Handbook of Clinical Neurology; Elsevier B.V., 2018; Vol. 157, pp. 565-597.

4. Mota, C.M.D.; Antunes-Rodrigues, J.; Branco, L.G.S. Central fractalkine stimulates central prostaglandin E 2 production and induces systemic inflammatory responses. Brain Res. Bull. 2018, 140, 311-317, doi:10.1016/J.BRAINRESBULL.2018.05.024.

5. Surendran, S.; Qassadi, F.; Surendran, G.; Lilley, D.; Heinrich, M. Myrcene-What Are the Potential Health Benefits of This Flavouring and Aroma Agent? Front. Nutr. 2021, 8, doi:10.3389/fnut.2021.699666.

6. Emílio-Silva, M.T.; Mota, C.M.D.D.; Hiruma-Lima, C.A.; Antunes-Rodrigues, J.; Cárnio, E.C.; Branco, L.G.S.S. Antipyretic Effects of Citral and Possible Mechanisms of Action. Inflammation 2017, 40, 1735-1741, doi:10.1007/s10753-017-0615-4. 